\title{
Van der Waals density functional: an appropriate exchange functional
}

\author{
Valentino R. Cooper ${ }^{1, *}$ \\ ${ }^{1}$ Materials Science and Technology Division, Oak Ridge, TN 37830-6114, USA
}

(Dated: May 30, 2018)

\begin{abstract}
In this paper, an exchange functional which is compatible with the non-local Rutgers-Chalmers correlation functional (vdW-DF) is presented. This functional, when employed with vdW-DF, demonstrates remarkable improvements on intermolecular separation distances while further improving the accuracy of vdW-DF interaction energies. The key to the success of this three parameter functional is its reduction of short range exchange repulsion through matching to the gradient expansion approximation in the slowly varying/high density limit while recovering the large reduced gradient, $s$, limit set in the revised PBE exchange functional. This augmented exchange functional could be a solution to long-standing issues of vdW-DF lending to further applicability of density functional theory to the study of relatively large, dispersion bound (van der Waals) complexes.
\end{abstract}

PACS numbers: 31.15.E-, 71.15.Mb, 61.50.Lt

Van der Waals, or London dispersion, interactions have profound importance in bio-organic systems as well as many novel materials being investigated for energy applications. Despite the importance of these systems and applications, first principles simulations have been greatly lacking. The primary reason for this is the inability of traditional density functional theory exchangecorrelation functionals to account for long-ranged, van der Waals interactions. This has limited first-principles investigations to quantum chemical methods which, due to their computational expense, are only capable of modeling fragments of the true material; thus often overlooking some of the more salient features of these systems.

A promising solution to the problem of balancing speed and scalability with accuracy lies in the non-local correlation functional of the Rutgers-Chalmers collaboration, the aptly named van der Waals density functional (vdW-DF). 1, 2] This method includes long-range dispersion effects as a simple perturbation to the local density approximation correlation term and has been extremely successful in describing a diverse group of materials properties - ranging from molecules to bulk polymers and the adsorption of molecules to surfaces and within bulk materials. 3 . Recent developments show that self-consistency gives no appreciable differences in computed interaction energies [1, 2] and such a non-local functional can in fact be incorporated in an extremely efficient manner. [4] However, the overwhelming success of vdW-DF is marred by its consistent overestimation of intermolecular distances. 3] Analysis of various generalized gradient approximation exchange functionals $\left(\mathrm{GGA}_{\mathrm{x}}\right)$ indicate that traditional functionals are either too repulsive at short distances or incorrectly exhibit some "correlation" binding at larger distances. The standard functional used within the vdW-DF, the revised PerdewBurke-Erzenhoff functional (revPBE), [5] unfortunately gives too much repulsion at short distances. Replacing revPBE with Hartree-Fock (HF) exchange shows improvements in the inter-species separation distances ob- tained with vdW-DF but at the cost of over-binding, i.e. considerably larger interaction energies than obtained via $\operatorname{CCSD}(\mathrm{T}) .[6,7]$ Recent work suggests that for many dispersion bound materials the PW86 functional [8] most closely matches HF exchange. 9, 10] Similarly when applied with vdW-DF it also strongly overbinds (see Fig. (2).

In this paper, a $\mathrm{GGA}_{\mathrm{x}}$ that may be more suitable for use with the vdW-DF correlation functional is proposed. This functional is derived through the introduction of an enhancement factor which obeys two specific constraints: (i) matching to the gradient expansion approximation (GEA) [1] in the slowly varying/high density limit and (ii) a smooth asymptote to the upper bound empirically set in revPBE exchange. Initial results indicate dramatic improvements in vdW-DF separation distances while retaining the accuracy of this method for a range of systems. Most notable are improvements in the interaction energies and the intermolecular/interplanar separation distances obtained for S22 database structures 12 and graphite.

The general formula of a $\mathrm{GGA}_{\mathrm{x}}$ can be written as:

$$
E_{\mathrm{x}}^{\mathrm{GGA}}=\int d^{3} r n \epsilon_{\mathrm{x}}^{\mathrm{unif}}(n) F_{\mathrm{x}}(s),
$$

where $\epsilon_{\mathrm{x}}^{\mathrm{unif}}(n)$ is the exchange energy per particle in a uniform gas $\left(\epsilon_{\mathrm{x}}^{\text {unif }}(n)=-3 e k_{F} / 4 \pi\right.$ with $\left.k_{F}=\left[3 \pi^{2} n\right]^{1 / 3}\right)$ and $F_{\mathrm{x}}(s)$ is the enhancement factor which is a function of $s=\nabla n /\left(2 k_{F} n\right)$. This form of exchange ensures proper, uniform density scaling [13] where $F_{\mathrm{x}}(s)=1$ simply gives LDA exchange. In general, the enhancement factor is chosen such that $F_{\mathrm{x}}(0)=1$. Here we design an $F_{\mathrm{x}}(s)$ to fulfill two further criteria:

(i) To reduce the short range exchange repulsion, in the limit of $s \rightarrow 0$, i.e. for slowly varying/high densities, the functional approaches the GEA:

$$
F_{x}^{G E A}(s)=1+\mu s^{2}
$$




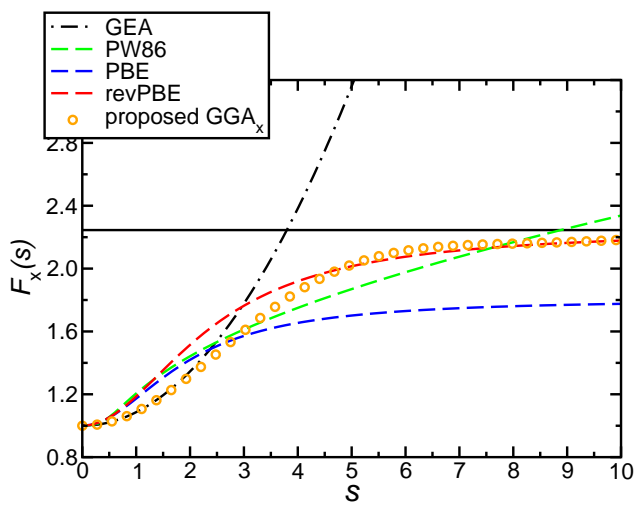

FIG. 1: (color online)Enhancement factor for various $\mathrm{GGA}_{x}$ functionals. The blue, red and green dashed lines represent the enhancement factor of PBE, [14] revPBE [5] and PW86, [8] respectively. The black, dashed-dot line is the GEA and the orange, open circles are points from the proposed C09 $x$. The solid, black line indicates the revPBE upper bound.

where $\mu=0.0864$. 11] Figure 1 depicts the enhancement factor of a number of $\mathrm{GGA}_{\mathrm{x}}$. It can be clearly seen that many of these functionals deviate quite quickly from the GEA. Note that this constraint is similar to that used in the recent PBEsol exchange functional which was designed to restore the gradient expansion in order to remove artificial bias towards free atoms. 15] Here, decreasing the enhancement factor for small $s$ (thus restoring the GEA) leads to a reduction in the short range repulsion in the $\mathrm{GGA}_{\mathrm{x}}$.

(ii) The second constraint used in the proposed $\mathrm{GGA}_{\mathrm{x}}$ is to asymptote the revPBE upper bound of 2.245 in the large $s$-limit. [5] This simple bound is taken to be compatible with previous applications of the revPBE exchange functional with the non-local vdW-DF correlation term. Here, we find that an empirical $F_{\mathrm{x}}(s)$ bound similar to revPBE gives the best interaction energies.

Using these constraints a simple, smooth, function can be constructed in the form:

$$
F_{\mathrm{x}}(s)=1+\mu s^{2} e^{-\alpha s^{2}}+\kappa\left(1-e^{-\alpha s^{2} / 2}\right)
$$

with $\mu=0.0617, \kappa=1.245, \alpha=0.0483$. Fig. 1 displays the enhancement factor of eqn. 3 along with that for other GGAs. The parameters were determined by simultaneously fitting Eqn. 3 to GEA for $s<1.5$ and to revPBE for $s>8.0$. This fitting domain was arbitrarily chosen to allow for a decrease in $F_{\mathrm{x}}(s)$ for small $s$ and a smooth recovery of revPBE for large values of $s$. (In accordance with previous naming conventions this functional shall be referred to as $\mathrm{C} 09_{x}$ ). The complimentary exchange potential can be easily constructed from the functional derivative of Eqn. 3 as shown in Eqn. 24 of Ref. 8.

To test the compatibility of the proposed $\mathrm{C} 09_{x}$ with vdW-DF, self-consistent calculations within a modified

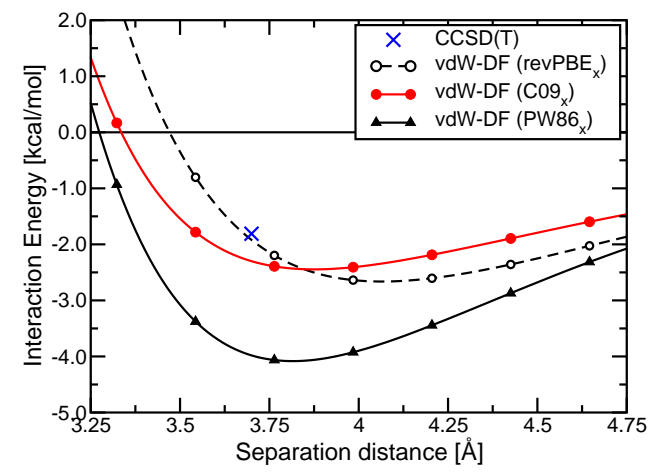

FIG. 2: (color online) Interaction energy as a function of separation distance for the benzene dimer in the stacked sandwich configuration. The vdW-DF results with revPBE, CO9 and PW86 for exchange are represented by a black dashed line with open circles, a red solid line with closed circles and a solid blue line with closed triangles, respectively. Benchmark $\operatorname{CCSD}(\mathrm{T})$ data [17] (blue cross) are plotted for reference.

version of the Abinit plane wave code [16 were performed. All calculations were carried out with a $30 \mathrm{Ha}$ planewave cutoff and a single k-point at $\Gamma$. To reduce the effects of periodic images, all simulation cells were padded with at least $10 \AA$ of vacuum in all directions.

The interaction energy, $\Delta E^{\text {int }}$, as a function of separation distance, $d_{\text {sep }}$, for the benzene dimer stacked in the sandwich configuration is plotted in Fig. 2, A comparison of vdW-DF with the standard revPBE exchange functional (vdW-DF ${ }^{\mathrm{revPBE}}$ ) and the exchange functional $\left(\mathrm{vdW}-\mathrm{DF}^{\mathrm{C} 09_{\mathrm{x}}}\right)$ using the enhancement factor of eqn. 3 shows a significant shortening of the separation distance from $4.07 \AA$ to $3.87 \AA$. The vdW-DF ${ }^{\mathrm{C} 09_{\mathrm{x}}}$ is now in much better agreement, with regards to both interaction energy and separation distance, with both the benchmark $\operatorname{CCSD}(\mathrm{T})\left(\Delta E^{\text {int }}=1.81 \mathrm{kcal} / \mathrm{mol}\right.$ and $\left.d_{\text {sep }}=3.70 \AA\right)$ as well as SAPT(DFT) $\left(\Delta E^{\text {int }}=1.67 \mathrm{kcal} / \mathrm{mol}\right.$ and $d_{\text {sep }}$ $=3.80 \AA)[24]$ calculations. As previously mentioned, the PW86 exchange functional, which was recently reported to mimic Hartree-Fock exchange for dispersion bound complexes, gives excellent separation distances, but significantly over estimates the interaction energy.

To further illustrate the value of the $\mathrm{C} 09_{\mathrm{x}}$, the interaction energy as a function of $c$ lattice parameter for graphite is plotted in Fig. 3. Here, C09 $\mathrm{x}$ offers significant improvements in both the value of graphite lattice constants as well the interplanar interaction energy. Our computed lattice constant of $6.56 \AA$ is within $2 \%$ of experiment $(6.70 \AA) .25]$ The interplanar binding energy $(-59 \mathrm{kcal} / \mathrm{mo})$ is also greatly improved $(-52 \pm 5 \mathrm{kcal} / \mathrm{mol}$ for experiment 25]). Note, $\mathrm{vdW}-\mathrm{DF}^{\mathrm{revPBE}} \mathrm{x}$ gives interaction energies and lattice constants of $-39 \mathrm{kcal} / \mathrm{mol}$ and $7.35 \AA$, respectively.

A more stringent evaluation of the accuracy of the functional can be gained through comparison with the benchmark S22 database of Jurecka et al.[12] This 
TABLE I: Computed interaction energies for the S22 model data set. 12] C09 values in parentheses are for full geometry optimizations. For comparison, vdW-DF interactions energies using the revPBE and PBE exchange functionals taken from Ref. 18 (unless otherwise noted) are listed. Deformation energies are not included. Energies are in kcal $/ \mathrm{mol}$.

\begin{tabular}{|c|c|c|c|c|c|}
\hline \multirow{2}{*}{ No. } & \multirow{2}{*}{ Complex } & \multicolumn{3}{|c|}{$\mathrm{vdW}-\mathrm{DF}$} & \multirow{2}{*}{$\begin{array}{c}\text { Benchmark } \\
\text { CCSD }(\mathrm{T}) / \mathrm{CBS}\end{array}$} \\
\hline & & $\operatorname{revPBE}^{a}$ & $\mathrm{PBE}^{a}$ & $\mathrm{C} 09$ & \\
\hline \multicolumn{6}{|c|}{ Hydrogen bonded complexes } \\
\hline 1 & $\left(\mathrm{NH}_{3}\right)_{2}\left(C_{2 \mathrm{~h}}\right)$ & 2.44 & 3.71 & $2.88(2.92)$ & 3.17 \\
\hline 2 & $\left(\mathrm{H}_{2} \mathrm{O}\right)_{2}\left(C_{\mathrm{s}}\right)$ & 4.08 & 5.58 & $4.99(5.15)$ & 5.02 \\
\hline 3 & Formic acid dimer $\left(C_{2 \mathrm{~h}}\right)$ & 14.07 & 18.28 & $20.12(24.59)$ & 18.61 \\
\hline 4 & Formamide dimer $\left(C_{2 \mathrm{~h}}\right)$ & 12.50 & 16.10 & $16.15(17.15)$ & 15.96 \\
\hline 5 & Uracil dimer $\left(C_{2 \mathrm{~h}}\right)$ & 16.17 & 20.59 & $20.93(22.36)$ & 20.65 \\
\hline 6 & 2 -pyridoxine $\cdot 2$-aminopyridine $\left(C_{1}\right)$ & 14.02 & 17.94 & $17.67(19.66)$ & 16.71 \\
\hline 7 & Adenine $\cdot$ thymine $\mathrm{WC}\left(C_{1}\right)$ & $15.19^{b}$ & 17.57 & $17.25(19.16)$ & 16.37 \\
\hline \multicolumn{6}{|c|}{ Complexes with predominant dispersion contribution } \\
\hline 8 & $\left(\mathrm{CH}_{4}\right)_{2}\left(D_{3 \mathrm{~d}}\right)$ & 0.88 & 1.55 & $0.51(0.51)$ & 0.53 \\
\hline 9 & $\left(\mathrm{C}_{2} \mathrm{H}_{4}\right)_{2}\left(D_{3 \mathrm{~d}}\right)$ & 1.41 & 2.68 & $1.16(1.16)$ & 1.51 \\
\hline 10 & Benzene $\cdot \mathrm{CH}_{4}\left(C_{3}\right)$ & $1.57^{c}$ & 2.51 & $1.50(1.71)$ & 1.50 \\
\hline 11 & Benzene dimer $\left(C_{2 \mathrm{~h}}\right)$ & $2.74^{d}$ & 4.96 & $3.32(3.40)$ & 2.73 \\
\hline 12 & Pyrazine dimer $\left(C_{\mathrm{s}}\right)$ & 3.87 & 6.25 & $4.74(4.75)$ & 4.42 \\
\hline 13 & Uracil dimer $\left(C_{2}\right)$ & $9.41^{e}$ & 12.91 & $10.31(10.41)$ & 10.12 \\
\hline 14 & Indole $\cdot$ benzene $\left(C_{1}\right)$ & 4.34 & 6.25 & $5.44(5.48)$ & 5.22 \\
\hline 15 & Adenine $\cdot$ thymine stack $\left(C_{1}\right)$ & $10.60^{e}$ & 14.74 & $12.79(12.73)$ & 12.23 \\
\hline \multicolumn{6}{|c|}{ Mixed complexes } \\
\hline 16 & Ethene $\cdot$ ethine $\left(C_{2 \mathrm{v})}\right)$ & 1.55 & 2.38 & $1.61(1.60)$ & 1.53 \\
\hline 17 & Benzene $\cdot \mathrm{H}_{2} \mathrm{O}\left(C_{\mathrm{s}}\right)$ & $2.72^{f}$ & 4.15 & $3.25(3.24)$ & 3.28 \\
\hline 18 & Benzene $\cdot \mathrm{NH}_{3}\left(C_{\mathrm{s}}\right)$ & 1.87 & 3.18 & $2.28(2.27)$ & 2.35 \\
\hline 19 & Benzene $\cdot \operatorname{HCN}\left(C_{\mathrm{s}}\right)$ & 3.87 & 5.488 & $4.51(4.48)$ & 4.46 \\
\hline 20 & Benzene dimer $\left(C_{2 \mathrm{v}}\right)$ & $2.05^{d}$ & 3.98 & $2.85(2.84)$ & 2.74 \\
\hline 21 & Indole $\cdot$ benzene $\mathrm{T}$-shape $\left(C_{1}\right)$ & 4.72 & 6.90 & $5.75(5.71)$ & 5.73 \\
\hline 22 & Phenol dimer $\left(C_{1}\right)$ & 5.81 & 8.51 & $7.00(7.20)$ & 7.05 \\
\hline Avg. & eviation & 18 & 36 & $5(9)$ & --- \\
\hline
\end{tabular}

${ }^{a}$ structures optimized for separation distance

${ }^{b}$ Ref. 19

${ }^{c}$ Ref. 20

${ }^{d}$ Ref. 6

${ }^{e}$ Ref. 22

${ }^{f}$ Ref. 23

database contains the interaction energies and structures of 22 structures with varying degrees of hydrogen bonding and vdW interactions computed with $\operatorname{CCSD}(\mathrm{T})$ extrapolated to the complete basis set limit and is currently accepted as the gold standard for theoretical methods used to study systems with significant dispersion interactions. Recently, Gulans and coworkers examined the S22 database using vdW-DF with both the revPBE and PBE exchange functionals. [18] In general, they found reasonable agreement with the interaction energies of the S22 database; with PBE producing better results for hydrogen bonded complexes and revPBE showing less deviation for dispersion dominated interactions. However, there results were all for vdW-DF optimized structures; which always give too large separation distances.
Table I lists the computed interaction energies for the S22 database. Here, it is evident that across the board vdW-DF $\mathrm{C}^{\mathrm{x}} \mathrm{x}$ is in much better agreement with the $\operatorname{CCSD}(\mathrm{T})$ benchmark values than when vdW-DF is used with either the PBE or revPBE functionals. In fact, $\mathrm{vdW}-\mathrm{DF}^{\mathrm{C} 09_{\mathrm{x}}}$ has an average percent deviation of $5 \%$ ( $9 \%$ if full geometry optimizations were performed); far less than revPBE and $\mathrm{PBE}$ ( $17 \%$ and $36 \%$, respectively). Even more important is the fact that these interaction energies were obtained using the published S22 geometries - without any adjustment of dimer separation distances, demonstrating once again the improvement that this functional offers with regards to both interaction energies and determining optimum separation distances.

All 22 structures were subsequently relaxed such that 


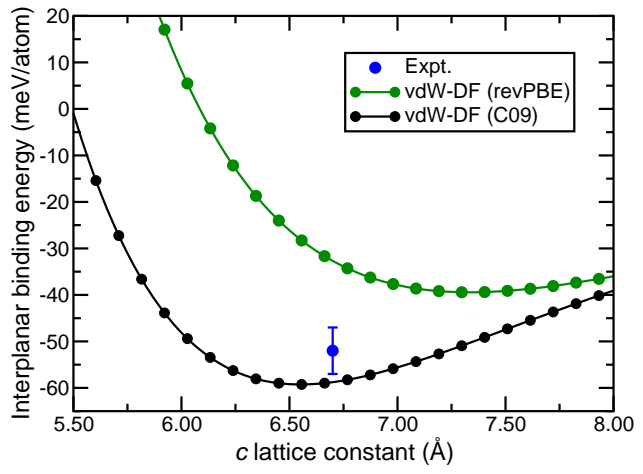

FIG. 3: (color online) Interaction energy as a function of interplanar separation distance for graphite. The vdW-DF results with revPBE and $\mathrm{CO} 9$ for exchange are represented by a black dashed line with open circles and a solid black line with closed triangles, respectively. A recent experimental value 25] (blue open circles with error bars) is plotted for reference.

the forces on all the atoms were less than $0.02 \mathrm{eV} / \AA$. These values are listed in parentheses in the Table I Analysis of the relaxed geometries indicate that the majority of the deviations are related to changes in internal bond lengths. This is evident in the larger changes in the $\mathrm{vdW}-\mathrm{DF}^{\mathrm{C} 09_{\mathrm{x}}}$ interaction energies for hydrogen-bonded structures.

In summary, an exchange functional that is compatible with the Rutgers-Chalmers van der Waals correlation functional is proposed. This functional was derived to closely match the enhancement factor $F(s)$ of the gradient exchange approximation for values of $0<\mathrm{s}<$ 1.5 , while having an asymptote to the revPBE bound of 2.245. In general, this functional shows significant improvements over the previous revPBE exchange. In particular, vdW-DF ${ }^{\mathrm{C} 09_{\mathrm{x}}}$ offers better agreement with the benchmark S22 database with an average deviation of only $5 \%$ at the intermolecular separation distances of the published geometries. This is a feat which far surpasses that of previous vdW-DF calculations which required larger separation distances. It should be noted that as previously pointed out that while the restoration of the gradient expansion gives improved dispersion interactions it is expected to worsen atomization energies. 15. Nevertheless, these results highlight the promise of this functional for use with the vdW-DF method and may offer a pathway to even more accurate first-principles calculations of dispersion bound systems.

VRC would like to thank David C. Langreth for many valuable discussions. VRC also acknowledges comments from Kieron Burke and discussions with Alaska Subedi. This work was supported by U.S. Department of Energy, Division of Materials Sciences and Engineering. This research used resources of the National Energy Research Scientific Computing Center, which is supported by the Office of Science of the U.S. Department of Energy under
Contract No. DE-AC02-05CH11231.

Electronic address: coopervr@ornl.gov

[1] M. Dion, H. Rydberg, E. Schröder, D. C. Langreth, and B. I. Lundqvist, Phys. Rev. Lett. 92, 246401 (2004).

[2] T. Thonhauser, V. R. Cooper, S. Li, A. Puzder, P. Hyldgaard, and D. C. Langreth, Phys. Rev. B 76, 125112 (2007).

[3] D. C. Langreth, B. I. Lundqvist, S. D. Chakarova-Kack, V. R. Cooper, M. Dion, P. Hyldgaard, A. Kelkkanen, J. Kleis, L. Z. Kong, S. Li, et al., J. Phys.: Condens. Matter 21, 084203 (2009).

[4] G. Román-Pérez and J. M. Soler, Phys. Rev. Lett. p. (in press) (2009).

[5] Y. Zhang and W. Yang, Phys. Rev. Lett. 80, 890 (1998).

[6] A. Puzder, M. Dion, and D. C. Langreth, J. Chem. Phys. 124, 164105 (2006).

[7] O. A. Vydrov, Q. Wu, and T. Van Voorhis, J. Chem. Phys. 129, 014106 (2008).

[8] J. P. Perdew and Y. Wang, Phys. Rev. B 33, 8800 (1986).

[9] F. O. Kannemann and A. D. Becke, J. Chem. Theory Comput. 5, 719 (2009).

[10] E. D. Murray, K. Lee, and D. C. Langreth, J. Chem. Theory Comput. (2009), doi: 10.1021/ct900365q.

[11] L. J. Sham, Computational Methods in Band Theory (Plenum, New York, 1971).

[12] P. Jurečka, J. Šponer, J. Černý, and P. Hobza, Phys. Chem. Chem. Phys. 8, 1985 (2006).

[13] M. Levy and J. P. Perdew, Phys. Rev. A 32, 2010 (1985).

[14] J. P. Perdew, K. Burke, and M. Ernzerhof, Phys. Rev. Lett. 77, 3865 (1996).

[15] J. P. Perdew, A. Ruzsinszky, G. I. Csonka, O. A. Vydrov, G. E. Scuseria, L. A. Constantin, X. Zhou, and K. Burke, Phys. Rev. Lett. 100, 136406 (pages 4) (2008).

[16] X. Gonze, J.-M. Beuken, R. Caracas, F. Detraux, M. Fuchs, G.-M. Rignanese, L. Sindic, M. Verstraete, G. Zerah, F. Jollet, et al., Comp. Mat. Sci. 25, 478 (2002).

[17] S. Tsuzuki, K. Honda, M. Mikami, and K. Tanabe, J. Am. Chem. Soc. 124, 104 (2002).

[18] A. Gulans, M. J. Puska, and R. M. Nieminen, Phys. Rev. B 79, 201105(R) (2009).

[19] V. R. Cooper, T. Thonhauser, A. Puzder, E. Schröder, B. I. Lundqvist, and D. C. Langreth, J. Am. Chem. Soc. 130, 1304 (2008).

[20] J. Hooper, V. R. Cooper, T. Thonhauser, N. A. Romero, F. Zerilli, and D. C. Langreth, ChemPhysChem 9, 891 (2008).

[21] V. R. Cooper, T. Thonhauser, and D. C. Langreth, J. Chem. Phys. 128, 204102 (2008).

[22] S. Li, V. R. Cooper, T. Thonhauser, B. I. Lundqvist, and D. C. Langreth, J. Phys. Chem. B p. 11166 (2009).

[23] S. Li, V. R. Cooper, T. Thonhauser, A. Puzder, and D. C. Langreth, J. Phys. Chem. A 112, 9031 (2008).

[24] R. Podeszwa and K. Szalewicz, Chem. Phys. Lett. 412, 488 (2005).

[25] R. Zacharia, H. Ulbricht, and T. Hertel, Phys. Rev. B 69, 155406 (2004). 